\title{
EFFECT OF MICRO-PROPAGATION ON THE SAPONIN CONTENT IN
}

\section{THE CHLOROPHYTUM}

\author{
A. S. Pandey \\ Department of Chemistry, Priyadarshini Institute of Engineering and \\ Technology, Nagpur
}

\begin{abstract}
Chlorophytum (Safed Musli) is a medicinal plant that grows in dense forests. It belongs to Liliaceae, a family of 175 species distributed all over the world. Among the 13 species found in India. It is a medicinal plant used to overcome weakness. It is a wellknown ayurvedic medicine rich in alkaloids, vitamins, minerals, protein, carbohydrate, steroids and polysaccharides. Dried roots of Chlorophytum contain $42 \%$ carbohydrate, $8-9 \%$ protein, $3-4 \%$ fiber and $2-17 \%$ saponin. Saponins are natural surfactants, or detergents, found in many plants.

The biotechnological research is multidisciplinary and acquires expertise of different areas. Research consists of characterization and testing of different proveniences and genotypes, development of suitable multiplication methods for plants, plant cells or tissues, either in the field, in micro propagation conditions or in bioreactors. The techniques of plant tissue culture offers means for mass multiplication, for biomass energy production as well as for the conservation of important, elite and rare species that are threatened in nature with danger of extinction . Exploitation of already existing variability in available germplasm is very important to identify superior genotype for local condition. Chlorophytum, although an important medicinal plants, but it has not received much attention from tissue culturist and only a few reports are available on in-vitro multiplication. In this study the effect of in-vitro regeneration on the saponin content was observed. The results of the study clearly demonstrates the reliability and enormous scope of in-vitro regeneration technology, but with due consideration for the specific genotype.
\end{abstract}

\section{Introduction:}

The success of the drug discovery process is often a function of the diversity of chemotypes examined. Natural products screening represents a potential source of organic chemicals of unparallel diversity. The screening of natural products is one of the earliest steps in drug 
discovery-Lead identification. A lead compound, also frequently referred to as a chemical template, is a compound with many of the characteristics of a desired new drug which will be used as a model for chemical modification, but which lacks either the potency or specificity expected of a product candidate. Historically, medicinal plants and microorganisms have been extraordinarily rich sources of medicinally and agriculturally useful compounds. Interest in these sources of new bioactive molecules continues to present time.

Medicinal plants comprise a group of large number of plant species that produce raw material for pharmaceuticals and phyto-chemicals for manufacturing drugs. In the commercial market, medicinal herbs are used as raw drugs, extracts or tinctures. The World Health Organization (WHO) estimates that up to $80 \%$ of the world population rely on plants for their primary health care. The international medicinal plants market is worth US $\$ 60$ billion per year, and growing at the rate of $7 \%$ per annum (Bhojvaid, 2003). Plants have contributed more than 7,000 different compounds in use as heart drugs, laxatives, diuretics, antibiotics, decongestants, analgesics, anesthetics, ulcer treatments antiparasitic compounds and so on (Ved et al., 1998). For the last few decades, phytochemistry (study of plants) has been making rapid progress and herbal products are becoming popular. A complete understanding of medicinal plants involves a number of disciplines including commerce, botany, horticulture, chemistry, enzymology, genetics, quality control and pharmacology.

The biotechnological research is multidisciplinary and acquires expertise of different areas. Research consists of characterization and testing of different proveniences and genotypes, development of suitable multiplication methods for plants, plant cells or tissues, either in the field, in micro propagation conditions or in bioreactors. The techniques of plant tissue culture offers means for mass multiplication, for biomass 
energy production as well as for the conservation of important, elite and rare species that are threatened in nature with danger of extinction (Jang et al., 2003). Plant tissue culture techniques are now being used also for monitoring of their secondary metabolites. The production of secondary metabolites by tissue culture has commercial potential as well as being useful in studying the biosynthesis and regulation of secondary products.

Safed Musli (Chlorophytum) is a medicinal plant that grows in dense forests. It belongs to Liliaceae, a family of 175 species distributed all over the world. Among the 13 species found in India, Safed Musli (Chlorophytum biovirilianum) has market potential (Borodia et al., 1995). Safed Musli is a medicinal plant used to overcome general and sexual weakness. It is a well-known ayurvedic medicine rich in alkaloids, vitamins, minerals, protein, carbohydrate, steroids and polysaccharides. Owing to its enormous uses, its worldwide demand is estimated to be 35000 tones annually as compared to current annual production of 5000 ton (Udhyamita samachar patra, 1998). Dried roots of Chlorophytum contain $42 \%$ carbohydrate, $8-9 \%$ protein, 3-4\% fiber and 2-17\% saponin (Borodia et al., 1995). The potential of plant tissue culture both source of high value chemicals and as a system for studying secondary metabolism has not yet been exploited (Holden et al., 1987). Chlorophtum (Safed musli) is endangered and rare medicinal plants of this state have been selected for in-vitro regeneration.

\section{Materials and Methods:}

Plantlets having well developed shoots with roots were transferred first to the sterilized sand. After one week, these plants were transferred to small pots, which contain a mixture of soil + compost + sand at the ratio of $1: 1: 1$ subsequently the plants acclimatized to semi shade condition before ultimately shifted to the field. Micropropogated plants grown in the field were compared with conventionally propagated for 
various morphological and biochemical characters. The morphological characters were recorded 8 months after transplanting during the field experimentation during year 2003 and 2004. Further the biochemical estimation was done after maturity.

\section{Results:}

\section{Assessment of in-vitro regenerated plantlets of Chlorophytum}

In-vitro regeneration in standardized medium was tested for all collected genotypes of Chlorophytum. All genotypes did not respond similar in the standardized media, which indicated that in-vitro potential was genotype specific. Micropropogated plants were field evaluated and compared with conventionally grown plants. Increase in number of tubers, length of solitary scape, leaf breadth, number of leaves were recorded in in-vitro regenerated plantlets of $\mathrm{M} 1$ in comparison with conventionally grown plants of M1. In contrary, comparatively less number of tubers, number of leaves, number of flowers, plant height, leaf breadth, leaf length and yield was recorded in in-vitro regenerated plantlets of M2. It has been found that all the phenotypic characters showed variation due to genetic and environmental interaction. The saponin content recorded were comparatively low in in vitro regenerated plantlets of Chlorophytum genotypes. The saponin content in in-vitro regenerated plants was $25.65 \%$ as compared to conventional grown, M1 genotype, which was $28.28 \%$. Similarly, in in-vitro regenerated plants of M2 it was $9.1 \%$ as compared to conventional grown M2, which was $10.75 \%$. This clearly indicated that micropropagation is not very effective in enhancement of saponin content, but it is very useful to break the dormancy and to get disease free planting material in large amount throughout the year.

In order to assess the in-vitro regeneration potential of Cholorophytum genotypes, and to produce large number of somaclones, high frequency in-vitro regeneration protocol was developed initially using 
two musli genotypes and testing of different explants were made. Large number of combinations and concentrations of phytohormones were tried; MS media supplemented with $5.0 \mathrm{mg} / 1$ BAP produced best shooting. Healthy roots were regenerated when shoots were transferred to half strength MS medium supplemented with $500 \mathrm{mg} / 1$ activated charcoal. After standardization of the in-vitro regeneration protocol, the potential of all Chlorophytum genotypes was observed and large number of soma clones of genotypes were produced and transferred to field for further evaluation of various traits including the quantity of lead compound. The in-vitro regenerated plantlets were studied and characterized at morphological and biochemical level. No significant variations were observed in the in-vitro regenerated plants with compare to conventionally grown plants.

\section{References:}

Bhojvaid, P. P. (2003). Medicinal Plants based Forest Management: Problems and Prospects. Indian Forester. 129: 25-36.

Borodia, P. C., Joshi, A. and Simlot, M. M. (1995). Safed Musli. Advances in Horticulture Vol. 11-Medicinal and Aromatic Plants. 428-449.

Holden, P. R., Holden, M. A. and Yeoman, M. M. (1987). Variation in the Secondary Metabolism of Cultured Plant Cells. Genetic Manipulation of Antibiotic Production. 14-26.

Jang, G. W., Kim, K. S. and Park, R. D. (2003). Micropropagation of Venus Fly Trap by Shoot Culture. Plant Cell and Organ Culture. 72: 95-98.

Udhyamita Samachar Patra. (1998). Safed musli. 13-22.

Ved, D.K., Barve, V., Begum, N. and Latha, R. (1998). Eco-Distributing Mapping of the Priority Medicinal Plants of Southern India. Current Science, 75(3):205-208. 